\title{
La educación socioemocional: su pertinencia en la formación y ejercicio profesional del psicólogo
}

\section{Socio-emotional education: relevance in the training and professional practice of the psychologist}

Recibido 18/10/2021

\author{
Eleyda Victoria Parada Treminio \\ Candidata a doctora en Educación, con \\ especialidad en Educación Superior, Universidad de \\ El Salvador \\ eleyda.parada@ues.edu.sv \\ https://orcid.org/0000-0001-6657-2237
}

\section{Referencia}

Parada Treminio, E. V. (2021). La educación socioemocional: su pertinencia en la formación y ejercicio profesional del psicólogo. Revista Guatemalteca de Educación Superior, 5(1), 118-126. https://doi.org/10.46954/revistages.v5i1.79

\section{Resumen}

OBJETIVO: reflexionar sobre la formación del profesional de la psicología, sus antecedentes, formación actual, los requerimientos del perfil, alcances y limitaciones identificadas, las implicaciones de estas en el desempeño laboral, y de qué forma pueden ser solventadas. MÉTODO: a partir del análisis documental realizado. RESULTADOS: se logró identificar estudios relacionados a la formación, el cual fue marcado por la tendencia de la psicología a nivel de Latinoamérica, estuvo centrada en formar un psicólogo general, que dentro de los planes de estudio de la universidad, se identifica en los 
contenidos la influencia de la filosofía, medicina y la educación, que el programa está centrado a la formación científica y técnica, que responde a la esfera cognitiva, pero el área socioemocional, ha estado desatendida en la formación, marcando la diferencia en el desempeño laboral. CONCLUSIÓN: las investigaciones consultadas brindan un panorama sobre las diferentes áreas por especialidad profesional, nivel educativo y desempeño en el mundo laboral indicando que favorecen el desempeño laboral, académico, social; para ello establecen propuestas para el desarrollo de una educación socioemocional que facilite el fortalecimiento, mejora o adquisición de las capacidades y habilidades. Así mismo reconocen que deben estar articuladas con el currículo y con una pedagogía para su formación.

OBJECTIVE: Reflect on the Professional formation of psychology, its history, profile requirements, scopes and limitations identified, implications of these on work performance, and the way that can be solved. METHOD: Based on the documentary analysis carried out. OUTCOMES: we were able to identify studies related to formation. which was marked by the trend of psychology at the Latin American level, which was to form a general psychologist within the study plans of the university, can be identified in the contents the influence of philosophy, medicine and the education, that the program is focused on scientific and technical formation which responds to the cognitive sphere but the socioemotional area have been unattended on the formation but the socioemotional area have been unattended on the formation, been the difference in the work performance. CONCLUSIONS: Researches consulted provides an overview of the different areas by professional specialty, educational level and performance in the workplace, indicating that they favor work, academic and social performance; For this purpose, establish proposals for the development of a socioemotional education that facilitates the strengthening, improvement or acquisition of capacities and skills. They also recognize that they must be articulated with the curriculum and with a pedagogy for their formation.
Palabras clave:

formación, profesional de la psicología, educación socioemocional.
Keywords: formation, psychology professionals, socialemotional education. 


\section{Introducción}

La educación socioemocional, pertinencia en la formación y ejercicio profesional del psicólogo, se plantea a partir de identificar en el proceso académico de los estudiantes de la carrera de licenciatura en psicología inscritos en la Facultad Multidisciplinaria Oriental, de la Universidad de el Salvador, la poca preparación recibida para el desempeño de algunas tareas que requieren poner en práctica de habilidades y capacidades socioemocionales en determinado momento. Algunos estudiantes han expresado verbalmente o por escrito, cierto grado de dificultad en la ejecución de las actividades prácticas de asistencia y/o intervención terapéutica, observándose también poca confianza en sí mismos y en sus capacidades para cumplir con lo que se le ha indicado en la tarea. Es de reconocer también que el plan de estudios está centrado en el conocimiento científico-técnico, no en el entrenamiento de habilidades socioemocionales. Es por ello que se observa la necesidad de incorporar una educación socioemocional en los estudiantes pues de acuerdo a lo investigado favorece al ejercicio profesional, que en el caso de los psicólogos es esperable y deseable en el mundo laboral. Este ensayo forma parte de una de las tareas investigativas relacionadas a profundización del tema de la tesis doctoral en educación.

\section{Contenido}

En los antecedentes consultados sobre el ejercicio profesional del psicólogo, se observa la vinculación estrecha entre la formación académica recibida y el desempeño laboral mostrado; como una forma de dar cuenta de la preparación académica alcanzada en el área científica, como en la personal. Dado esa correspondencia entre ellas se realiza un abordaje general sobre el surgimiento de la formación de psicología en El Salvador desde la academia, sus inicios, el plan de estudios, entre otros.

La Universidad de El Salvador inició la formación de profesionales de psicología, posterior a la presencia de esta como disciplina académica, en un primero momento apareció como contenido de cátedra de Filosofía que era impartida desde 1846; aunque no de una forma profunda, ni especializada (Portillo, 2006). Esto 
hecho se debe a la tendencia que la psicología marcaba en ese momento en Latinoamérica, que de acuerdo a Rubén Ardila (1969), esta surge de tres fuentes principales: la filosofía, la educación y la medicina. En el caso de El Salvador, la psicología se desarrolló de las mismas fuentes, en lo que respecta a la medicina se visibiliza su mayor aporte en la construcción del hospital psiquiátrico y en investigaciones publicadas relacionadas al tema de salud mental; en lo que respecta a educación, fue el gabinete pedagógico que evidenció los requerimientos de la psicología en este campo.

Fue así como en el año 1956 el Departamento de Psicología inicia sus labores, se impartieron en el primer año la cátedra de Psicología Experimental, Introducción al Psicodiagnóstico de Rorschach, Psicoanálisis y Estadística; Aplicación de Pruebas Psicológicas, Mediciones Mentales y las Prácticas; como se puede apreciar el plan de estudios se encontraba centrado en formar al profesional con conocimiento técnico, no en contenidos socioemocionales o actitudinales, es decir no se observa una formación integral. La duración del plan de estudios en ese entonces, era de dos años y el grado a obtener era de Psicómetra, si se quería optar por ser psicólogo consultor, escolar o industrial se debía realizar dos años más de estudios.

La formación que la Universidad de El Salvador desarrolla mediante el plan de estudios vigente de la carrera de Psicología es del año 1998, se orienta a la formación de un psicólogo general, con énfasis en la investigación científica y el ejercicio de una práctica psicológica dirigida a conocer y facilitar la resolución de problemáticas, alteraciones psicológicas en cualquier campo de la familia y la sociedad. (Universidad de El Salvador,2006). Considerando que esta tarea es muy amplia y compleja, se requiere de desarrollar transversalmente una pedagogía para la educación socioemocional.

En esta vía se identificó una investigación de pregrado de la licenciatura de psicología, que tuvo como objetivo contrastar los conocimientos recibidos en la formación de los psicólogos, con los desafíos a los que se enfrentan en las empresas e instituciones donde laboran (Alvarado Sánchez, Canizales Ochoa, \& Medicina 
Romero, 2009). Participaron 45 profesionales de instituciones públicas y privadas, con roles de jefatura, empleados y en el ejercicio libre de la profesión.

Entre los resultados obtenidos destacan que, alto porcentaje de los participantes expresan no estar satisfechos con los conocimientos recibidos; que las habilidades adquiridas en la formación se encuentran a un nivel intermedio, siendo de vital importancia en el desempeño laboral, el nivel alcanzado no es suficiente y que se debe a que, en el proceso académico no fueron potenciadas y en consecuencia dificultan el ejercicio de la profesión. En este sentido es necesario prestar particular atención a este hallazgo, debido a las repercusiones que produce en el ejercicio profesional y, además, por ser un indicador que señala directamente que, la formación debe orientarse a fortalecer, incorporar, perfeccionar esas capacidades y habilidades, por lo tanto, se propone establecer una pedagogía de educación socioemocional.

Los resultados de diferentes investigaciones que indican que el desarrollo de las competencias transversales en educación superior es posible y necesario para la formación de profesionales cualificados, (Pérez-Escoda, Berlanga Silvente, \& Alegre Rosello, 2019). Así también Pertegral-Felices, CastejónCosta \& Martínez, (2011), sostiene que es necesario que desde la currícula universitaria se promueva el desarrollo de competencias para un desarrollo profesional eficaz. Al respecto el informe Delors (1996), señala la urgente necesidad de que los sistemas educativos formales propenden a dar prioridad a la adquisición de conocimientos, en detrimento de otras formas de aprendizaje, importa concebir la educación como un todo. En consecuencia, a estas investigaciones se contempla una pedagogía socioemocional como vía a la formación integral del psicólogo.

Los estudios consultados sobre la formación de habilidades socioemocionales en los psicólogos, presentan una variedad de categorías asumidas en la investigación como lo es inteligencia emocional, educación emocional, habilidades sociales, competencias, habilidades socioemocionales, unas centradas 
en la emoción y su educabilidad y otras en el resultado de la interacción social. Algunos de ellos han sido realizados desde metodologías de investigación mixta, cuantitativa o cualitativas. Independientemente dela categoría y enfoque utilizado concluyen sobre su importancia, pertinencia y diferencia que marca en cualquiera de los niveles educativos, desde la primera infancia hasta en los estudios de posgrado donde se haya implementado un programa, una estrategia, una pedagogía o curso de formación.

Por otra parte, los estudios presentan las distintas áreas profesionales donde se ha investigado la presencia de la dimensión socioemocional en el desempeño laboral, y los resultados han sido concluyentes, indican que es importante el fortalecimiento de las capacidades socioemocionales, indistintamente del área sea esta de salud, educativa, empresarial, industrial y en cuál de las habilidades, capacidades, competencias se centren. En cuanto a cómo desarrollar las competencias emocionales en la educación superior, Souto Romero (2012) reconoce que hay dificultades para articular las competencias del curriculo universitario con las que demanda el mundo laboral, se desconoce como se incorporará al curriculo, dado las barreras que se presentan.

Partiendo de lo anterior se considera que si bien es necesario que esté incorporado en el plan de estudios, es fundamental, pero, no lo suficiente, es necesario trasladar esa intención educativa planteada, a los modos de proceder docente ejecutándose desde una pedagogia sociemocional, que aborde con claridad la capacidad, habilidad o competencia sociemociconal a formar, sea esta intrapersonal o interpersonal, seguidamente como todo acto pedagógico requiere que sea planificado, debe declarar si es de formacióon directa, mediante modelacion o ejerccicio práctico y especificar, es decir porqué esa capacidad, habilidad y no otra.Las valoración de cual capacidad socemocional trabajar transversalmente en el programa académico, sea mediante estrategias de aprendizaje colaborativo o por otra metodologia vevencial. Para ello es necesario establecer el encuadre con la población estudiantil, pues en la formación universitaia se debe considerar tambien, la disposición del estudiante, si la persona muestra disposicón al proceso formativo o resistencia a él. 
En este sentido bien puede solventarse la situacion a través de una formación extracurricular que complemnete las unidades de aprendizaje exigidas en el programa académico, que se dimensione la disciplina, que en este caso es la psicologia y al sujeto de aprendizaje, sobre todo en los paises latinoamericanos como lo es El Salvador, que buena parte de la población inscrita en la carrera de psicologia, lleva una huella mnémica en su historia personal, comunitaria y social historias de muchas vulneraciónes, marcadas por pautas de interacción autoritarias, negligentes y poco asertivas. Desencadendo una interaccion social restrictiva, limitada y/o evitativa. Situación que los hace estar en alerta y respondiendo en algunos momentos de forma impropia o inesperada, hasta para ellos mismo.

La tarea en formación de capacidades y habilidades en los estudiantes de psicologia es permanente, pero a medida avanza debe ir intensificándose y profundizando en genericas y elementalmente básicas como lo son la escucha activa, empatia, comunicación asertiva; ahora bien para ser manifestadas con dominio alto, el estudiante previamente debe haber ejercitado en él mismo, escuchándose, conociéndose, identificando como responde a los acontecimientos, que emociones les suscita este u otro evento, las formas de refirse asi mismo, a qué le presta atención, si son emociones postivas y negativas, entre otros. Este proceso debe ser acompañado mediante un sistema de ayudas, sean estas de sus pares o de un tutor.Es decir pasar por un entrenamiento personal que le permita responder al ejercicio profesional no solo con conociemiento cientifico tecnico sino tambien desde el saber ser persona.

\section{Conclusión}

A partir de lo planteado, se logra identificar el estado real de la formación profesional del psicólogo de la Facultad Multidisciplinaria Oriental de la Universidad de El Salvador, requiere ser fortalecida en la dimensión socioemocional, mediante una pedagogía que favorezca la transición del aprendiz al a profesional, ejerciendo su rol con dominio, con capacidades y habilidades que el mundo laboral solicita, favoreciendo su dimensión personal, profesional, la empleabilidad y en lo que respecta a la universidad cumpliendo el encargo social exigido a la psicología como disciplina académica. 


\section{Referencias bibliográficas}

Alvarado Sánchez , V. L., Canizales Ochoa, A. G., \& Medicina

Romero, E. M. (agosto de 2009). La formación académica que brinda el Departamento de Psicología de la Universidad de El Salvador y su correspondencia con las competencias académicas y profesionales exigidas por empresas e instituciones del sector metropolitano de San Salvador; propuesta. San Salvador, El Salvador . https:// ri.ues.edu.sv/id/eprint/16329/1/14101598.pdf

Delors, J. (1996). "Los cuatro pilares de la educación"En la Educación encierra un tesoro. Madrid, España: Santillana / UNESCO. https://uom.uib.cat/digitalAssets/221/221918_9. pdf

Pérez-Escoda, N., Berlanga Silvente, V., \& Alegre Rosello, A. (2019). Desarrollo de Competencias Socioemocionales en Educación Superior: Evaluación del Posgrado en Educación Emocional. Bordón, Revista de Pedagogía, 97113. https://recyt.fecyt.es/index.php/BORDON/article/ view/64128

Pertegral-Felices, M. L., Castejón- Costa, J. L., \& Martínez, M. Á. (2011). Competencias Soioemocionales en la Formación del Maestro. Redalyc, 237-260. http://www.redalyc.org/ pdf/706/70618742010.pdf

Portillo, N. (2006). Antecedentes, desarrollo y aplicaciones tempranas de la psicología en El Salvador (1850-1950). Revista de Historia de la Psicología, 7-68. https://dialnet. unirioja.es/servlet/articulo?codigo $=2259139$

Souto Romero, M. d. (2012). Desarrollo de competencias emocionales en la educación superior. Dialnet. https://doi. org/10.13042/Bordon.2019.64128

Universidad de El Salvador. (2006). Plan de estudios de la carrera de la licenciatura en psicología. Plan de estudios de la carrera de la licenciatura en psicología. El Salvador. 


\section{Sobre la autora}

Eleyda Victoria Parada Treminio es Licenciada en Psicología, graduada en la Maestría en Métodos y Técnicas de Investigación Social de la Universidad de El Salvador. Es candidata a doctora en Educación, con especialidad en Educación Superior en la Universidad de El Salvador.

\section{Conflicto de intereses}

Declara no tener ningún conflicto de intereses.

\section{Declaración de consentimiento informado}

El estudio se realizó con fines educativos, respetando el Código de ética y buenas prácticas editoriales de publicación.

\section{Derechos de uso}

Copyright@ 2021 por Eleyda Victoria Parada Treminio

Usted es libre para compartir, copiar y redistribuir el material en cualquier medio o formato y adaptar el documento, remezclar, transformar y crear a partir del material para cualquier propósito, incluso comercialmente, siempre que cumpla la condición de atribución: usted debe reconocer el crédito de una obra de manera adecuada, proporcionar un enlace a la licencia, e indicar si se han realizado cambios. Puede hacerlo en cualquier forma razonable, pero no de forma tal que sugiera que tiene el apoyo del licenciante o lo recibe por el uso que hace. 